\title{
Caracterización del terroir: influencia en la composición de la uva y relación con el NDVI dentro de la D.O.Ca.Rioja I Terroir characterization: influence on grape composition and relationship with NDVI within D.O.Ca.Rioja
}

\author{
Álvaro Martínez ${ }^{1,2, a}$ y Vicente D. Gómez-Miguel ${ }^{2}$ \\ ${ }^{1}$ Bodegas de los Herederos del Marqués de Riscal. Ctra. Nacional VI, km 172.600 Rueda (Spain) \\ ${ }^{2}$ Departamento de Producción Agraria. Universidad Politécnica de Madrid (UPM), Spain
}

\begin{abstract}
Resumen. Los factores naturales que definen el terroir son los principales responsables de la variabilidad observada entre plantas de una misma parcela (variabilidad intraparcela) o de distintas fincas (variabilidad interparcelas). En el presente trabajo se propone una metodología de zonificación del terroir, presentándose también su relación con algunas características vegeto-productivas del viñedo y con el Índice de Vegetación de Diferencia Normalizada (NDVI). La zonificación de los elementos del medio se ha llevado a cabo a partir de la fotointerpretación aérea mientras que la representación gráfica del NDVI se realiza partiendo de una imagen multiespectral capturada por el satélite Pleiades, de 0,5 metros de resolución espacial. Comparando los datos vegeto-productivos recogidos en las 4 parcelas de estudio, se ha observado que existen sectores, relacionados con zonas de deposición de material y asociados a suelos de carácter fluvéntico, con tendencia a ser más vigorosos, más productivos y con uvas de menor contenido fenólico y de menor $\mathrm{pH}$. Por otro lado, analizando cada parcela de manera independiente, se observa que la distribución espacial del NDVI sigue un patrón semejante a la zonificación de los elementos del medio, algo que recomendaría hacer un uso de este índice por sectores previamente discriminados conforme a una cierta uniformidad del terroir.
\end{abstract}

\begin{abstract}
The natural factors that define terroir are main responsible for the variabililty observed between plants of the same plot (intra-plot variability) or of different farms (variability between plots). A methodology of terroir zoning is proposed, also is presented its relation with some vegeto-productive characteristics of vineyard and with the Normalized Difference Vegetation Index (NDVI). Zoning of natural factors has been carried out from aerial photointerpretation while graphic representation of NDVI was made starting from a multispectral image $(0,5$ meters of spatial resolution) captured by the Pleiades satellite. Comparing the vegetative and productive data collected in the four study plots, it has been observed that there are sectors related to deposition areas and associated with fluvent soils, with a tendency to be more vigorous, more productive and with grapes of lower phenolic content and lower $\mathrm{pH}$. On the other hand, analyzing each plot independently, it is observed that NDVI spatial distribution follows a similar pattern to the natural factors zoning, something that would recommend making use of NDVI by sectors previously discriminated according to certain terroir uniformity.
\end{abstract}

\section{Introducción}

Los primeros estudios científicos relacionados con los elementos del medio vitícola y sus interacciones se llevan a cabo en el último cuarto del siglo XX, pudiendo considerarse la tesis doctoral del profesor René Morlat (1) uno de los estudios pioneros sobre la zonificación del terroir vitícola en el sentido moderno del término. El citado trabajo se desarrolla en la zona media del valle del Loira, siendo los países europeos los que tradicionalmente más importancia han otorgado a los elementos del medio en la caracterización de los vinos, protegiendo así el origen de los mismos. Dos ejemplos de esta tradición son la clasificación actual de vinos de Burdeos, que prácticamente no ha sufrido modificaciones desde su creación en 1855 y la clasificación de vinos de Oporto que tiene su origen en la delimitación de 1758 (actualmente esa zonificación se ha visto ampliada) llevada a cabo por la Companhia Geral da Agricultura das Vinhas do Alto Douro (empresa similar a los actuales Consejos Reguladores) a propuesta del Marqués de Pombal.

La clasificación de los vinos en el marco de la D.O.Ca.Rioja data de 1970 y está basada en el 
envejecimiento de los mismos, existiendo las clases Garantía de Origen (o Genérico), Crianza, Reserva y Gran Reserva. Recientemente (junio de 2017), el Consejo Regulador de la D.O.Ca. Rioja ha aprobado la figura de "Viñedo Singular", que, entre otros aspectos, protege viñedos que se caracterizan por poseer una delimitación natural técnicamente justificable, una edad de las plantas superior a 35 años, y un rendimiento más de un $20 \%$ inferior al autorizado para el conjunto de la Denominación de Origen (D.O). Para una adecuada incorporación tanto de esta nueva protección como de hipotéticas nuevas figuras parece necesario el uso de metodologías de zonificación rigurosas y objetivas que permitan clasificar los diferentes terroir de la D.O., huyendo de potenciales métodos de caracterización subjetivos y poco exhaustivos que pondrían en riesgo su éxito.

\subsection{Terroir}

El concepto de terroir incluye, además de los elementos del medio vitícola, un factor enológico y un factor humano, presente en los dos anteriores (2). Después de la vendimia, el manejo de la cosecha queda subordinado a la enología, cuyos procesos han evolucionado notablemente en España desde los años 70 del siglo XX (3), mejorándose, entre otros aspectos, la higiene de las bodegas o el control de los procesos microbiológicos, térmicos, de envejecimiento, etc. Tomando, por lo tanto, el momento de la vendimia como punto de inflexión, podemos diferenciar entre un aspecto vitícola y otro vinícola dentro del terroir vitivinícola, sobre el primero gravita este trabajo.

El clima es un factor de gran importancia en la calificación de una región o zona en relación con la aptitud para producir uvas de calidad (4), siendo sus efectos notables en el nivel de maduración de una determinada variedad y en la tipicidad y la calidad del vino obtenido $(4,5)$. Para estudiar la influencia del clima en el viñedo es tradicional distinguir, en función de la escala, entre macroclima, mesoclima y microclima. El primero hace referencia al clima de una región y es el principal factor limitante para el cultivo de la vid (6), mientras que el mesoclima es característico de una ubicación topográfica y paisajística determinada y afecta a un conjunto de plantas por igual en una determinada unidad geomorfológica. Por último, el microclima se refiere al de la propia planta, rodea a hojas y racimos y tiene una gran influencia en el ciclo biológico (por ejemplo, es de gran importancia en el periodo de maduración de la uva), pudiéndose modificar, además, a través de las operaciones del cultivo.

Al igual que sucede con el clima, se producen vinos de alta calidad a partir de uvas cultivadas sobre geologías (estratigrafía y litología) diferentes. Según (7) la geología influye en tres factores que afectan a la calidad del vino: (i) modificando la temperatura alrededor de la planta y de los racimos; (ii) controlando el suministro de humedad y la velocidad en la toma de nutrientes; (iii) determinando la disponibilidad de nutrientes en el suelo. Respecto a este último punto, la geología es un factor importante en el control de la nutrición potásica de la vid (7). (8) apunta que la influencia directa de la geología sobre el viñedo es mínima en suelos profundos, afectando de forma indirecta en suelos superficiales, a través de la composición del suelo, la geomorfología y la capacidad de retención de agua. Por otro lado, el efecto de la geomorfología sobre el terroir es indirecto, a través de la altitud, la pendiente y la orientación o exposición $(9,10)$.

Una concepción tradicional del suelo permite considerarlo como el resultado de la interacción del clima y de los seres vivos, como factores activos, sobre el tipo de roca y el relieve, como factores pasivos, durante un tiempo de actuación determinado. Desde un punto de vista vitícola, el suelo es el sustentáculo de las plantas, el almacén de agua y nutrientes que estas utilizan y el regulador de los elementos del clima (temperatura y precipitación, por ejemplo) que actúan sobre ellas (6). De igual manera que sucedía con el clima, y lógicamente con la geología, se elaboran vinos de alta calidad a partir de uvas producidas sobre una gran diversidad de suelos, siendo imposible definir los mejores suelos en términos de pedregosidad, contenido en arcilla o limo, profundidad de suelo o contenido mineral (9). La descripción de suelos cultivados con viñedo ha estado durante mucho tiempo reducida a los tipos de textura, pero, por ejemplo, un mismo contenido en arcilla no tiene las mismas propiedades, ya que éstas dependen de la naturaleza mineralógica de la arcilla en cuestión. Así, las convicciones basadas en el empirismo popular se revelan a veces contradictorias debido a su carácter simplista (11).

La elección del portainjerto a utilizar y de la variedad a cultivar debe considerar las condiciones del medio en el que se implantará el viñedo, con el principal objetivo de conseguir una madurez adecuada de la uva. Varios autores $(4,12-14)$ discriminan entre zonas de maduración en función de distintos criterios.

Las distintas técnicas utilizadas para el cultivo de la vid y su influencia en la respuesta de la planta han sido, y son, objeto de numerosos estudios. Sin duda, las operaciones culturales (por ejemplo el sistema de conducción, el marco de plantación, la utilización y manejo del riego, la poda en verde, etc.) afectan, en distinta medida, al rendimiento de la cosecha y a la composición de la uva.

\subsection{Variabilidad vitícola}

Columela titula el capítulo primero del Libro III de De Re Rustica (15):'Que especie de vid convenga a cada terreno y a cada clima", donde habla de la versatilidad de la vid para adaptarse a distintos climas y suelos, y se dan indicaciones para elegir la variedad de vid adecuada según las características de estos dos factores. Las diferencias entre los factores naturales darán lugar a una variabilidad en las características del cultivo. Algunos de estos factores son el clima y el suelo $(16,17)$, la altitud, la pendiente y su orientación (18) y/o la geología $(8,16$, 19). En un estudio llevado a cabo en el municipio de Oyón, dentro de la D.O.Ca. Rioja, (20) encuentran como elementos del medio responsables de la variabilidad interparcela la altitud, la geología, el suelo, la pendiente y la orientación. 
Por otro lado, también es conocida la variabilidad existente en la maduración de las uvas dentro de la misma parcela (variabilidad intraparcela), así Cadet-de-Vaux en 1803 (21) señala: “...nunca se hará buen vino sin dos vendimias, porque nunca puede madurar igualmente el fruto". Más recientemente, (22) señalan que actualmente con ayuda de la Viticultura de Precisión (PV) se están realizando vendimias bien separando la uva vendimiada el mismo día en diferentes lotes, o bien vendimiando en días distintos cada zona de la parcela con el objetivo de obtener el mayor grado de calidad en cada bloque. (23) apunta que este tipo de vendimia es viable a distintas escalas de producción, desde pequeños hasta grandes elaboradores. Algunos de los factores del medio a los que puede deberse la variabilidad intraparcela son: (i) la topografía $(9,24)$; (ii) la profundidad del suelo (25); (iii) la capacidad de retención de agua del suelo $(22,26)$ y/o (iv) el contenido en nitrógeno del suelo $(27,28)$. Además, esta variabilidad tiene efectos tanto en el rendimiento (29) como en la calidad de la uva (30), siguiendo (en estos dos últimos ensayos) un patrón parecido año tras año.

En resumen, los factores naturales que definen el terroir son los principales responsables de la variabilidad observada entre plantas de una misma parcela (variabilidad intraparcela) o de distintas fincas (variabilidad interparcelas).

En el presente trabajo se propone una metodología de zonificación del terroir, así como su relación con algunas características vegeto-productivas del viñedo y con el Índice de Vegetación de Diferencia Normalizada (NDVI).

\section{Material y Métodos}

El ensayo se lleva a cabo en 4 parcelas (A, B, C, D) de viñedo, amparadas por la D.O.Ca. Rioja y certificadas en agricultura ecológica por el Consejo de Agricultura y Alimentación Ecológica de Euskadi, situadas en el municipio de Oyón.

Sobre estas parcelas se hace una zonificación bajo criterios vitícolas (variedad, sistema de conducción y edad del viñedo) y en las subparcelas resultantes se realiza una fotointerpretación aérea discriminando sectores (A1, A2, A3 para la parcela A y de forma análoga para el resto de parcelas). En cada sector se marcan 12 cepas, repartidas en 2 repeticiones de 6 plantas, sobre las que se realizarán análisis y medidas vegetativas (longitud del sarmiento, diámetro del sarmiento y peso de la madera de poda), productivas (número de racimos y peso de los mismos) y físicoquímicas de la uva vendimiada.

Según el Sistema Multicriterio de Clasificación Climática (MCCS) (5) el clima de la zona donde se enmarcan las parcelas es templado cálido $(\mathrm{HI}+1)$, de noches frescas $(\mathrm{CI}+1)$ y moderadamente seco $(\mathrm{DI}+1)$, situándose la mayoría de los viñedos de la zona sobre areniscas y lutitas de la Facies de Haro (Mioceno MedioSuperior). Algunos de los suelos encontrados sobre esta geología y su sistema cuaternario asociado son alfisoles (por ejemplo del subgrupo Haploxeralf Cálcico), entisoles (por ejemplo del subgrupo Xerofluvent Típico) o inceptisoles (por ejemplo del subgrupo Xerocrept Típico). Para más detalle de la zona de estudio ver (20).

Para el cálculo del índice de vegetación de diferencia normalizada (NDVI) se parte de una imagen multiespectral (incluyendo rojo e infrarrojo cercano) capturada por el satélite Pléiades el 19 de agosto de 2015, de 0,5 metros de resolución espacial. El cálculo y la representación gráfica del índice se lleva a cabo con el software ArcGIS 10.1 del Instituto de Investigación de Sistemas Medioambientales (ESRI). La definición de las clases (Muy baja, Baja, Media, Alta y Muy Alta) se realiza conforme a cinco cuantiles, correspondiéndose el primer cuantil con la clase Muy baja y el quinto cuantil con la clase Muy Alta. Con el objeto de conocer y poder operar con el valor del NDVI, se calculará el valor medio del índice dentro de cada sector.

El análisis estadístico de los datos se efectuó mediante Análisis de Componentes Principales (ACP), Análisis Factorial Discriminante (AFD) y ANOVA univariante, previa comprobación de normalidad y homogeneidad de varianzas de las variables. La significación de estos análisis se determinó para los niveles de probabilidad $\mathrm{p}<0.05\left(^{*}\right), \mathrm{p}<0.01(* *)$ y $\mathrm{p}<0.001(* * *)$. Las medias se comparan mediante el test de Duncan cuando hubo diferencias significativas en el análisis de varianza. Para los análisis ANOVA se utilizó el programa SPSS, versión 15.0 (SPSS Inc. Chicago, Illinois) y para el resto de cálculos estadísticos el complemento XLSTAT 2009.1.02 sobre Microsoft Excel 2007.

\section{Resultados y Discusión}

El clima en la zona de ensayo durante el año 2015, según la clasificación MCCS, fue templado cálido $(\mathrm{HI}+1)$, de noches muy frescas $(\mathrm{CI}+2)$ y sub-húmedo (DI1)

En las Tablas 1 y 2 se presentan los resultados obtenidos en cada sector según aspectos vegetoproductivos y físico-químicos, respectivamente.

Todos los parámetros relacionados con el crecimiento vegetativo y la producción (Tabla 1) han mostrado diferencias muy significativas. La parcela $\mathrm{C}$ (sectores $\mathrm{C} 1$ y C2) y el sector D2 aparecen como las zonas más productivas y de mayor peso de madera de poda, respecto a este último parámetro también el sector D3 es significativamente alto. Respecto a la variabilidad intraparcela, se observa que los sectores A2 y D2 (en mayor medida) presentan una tendencia más productiva y más vigorosa. Estos sectores se corresponden con zonas bajas de deposición de material, generalmente asociadas a suelos de carácter fluvéntico como los del grupo Xerofluvent.

Los parámetros relacionados con las características de la uva que presentan diferencias significativas (con distinto nivel de significación) son (Tabla 2): grado de alcohol probable, $\mathrm{pH}$, Índice de Color (I.C), Índice de Polifenoles Totales (I.P.T.), Antocianos, Peso de 100 bayas y Potasio. Destacan de nuevo los sectores C1, C2 y D2 por ser los de menor contenido fenólico (I.C., I.P.T. y antocianos), así como por un menor $\mathrm{pH}$, en particular el sector D2. Resultados similares han sido encontrados por 
(31) dentro del ámbito de la D.O.Ca.Rioja, en el mismo municipio de Oyón.

Por otro lado, la representación gráfica del NDVI (Figura 1) sigue una distribución que se ajusta a la división realizada con ayuda de la fotointerpretación aérea. Por lo tanto se puede afirmar que tanto a una escala entre parcelas (20) como a nivel intraparcela, los elementos del medio (altitud, geología, geomorfología,...) tienen influencia sobre el NDVI. Además, algunas de las características vegeto-productivas del viñedo (datos no mostrados), como el peso de madera de poda, se correlacionan en gran medida con el NDVI. Fijándonos en la parcela $\mathrm{D}$ de la Figura 1, se puede apreciar como el sector D2 está asociado a valores muy altos del NDVI y, como se ha visto más arriba, este sector se caracteriza por ser vigoroso, productivo, de bajo contenido fenólico y bajo pH. Esta influencia de los elementos del medio sobre el NDVI recomendaría hacer un uso de este índice por sectores previamente discriminados conforme a una cierta uniformidad del terroir.

Para entender mejor la fuente de la variabilidad en el comportamiento de la vid se hace necesario realizar un estudio de suelos con el detalle adecuado en función del tamaño de la parcela. La caracterización de los suelos de cada sector, así como otras características relacionadas con el terroir se pondrán de manifiesto en la tesis doctoral titulada "Caracterización e influencia del terroir sobre la composición de la uva y los componentes vegeto-productivos de la vid (Vitis vinifera L.) en la D.O. Ca. Rioja", que próximamente verá la luz y donde también se podrá contrastar la presencia de estos suelos en el ámbito de la D.O.Ca. Rioja.

\section{Conclusiones}

En el presente trabajo se ha podido comprobar que la variabilidad intraparcela, presente en los viñedos del ensayo, sigue un patrón definido por los elementos del medio a través de la fotointerpretación aérea. Los sectores correspondientes a zonas de deposición de material se asocian con viñedos vigorosos, productivos, con bajo contenido fenólico y bajo $\mathrm{pH}$.

A su vez, el NDVI sigue una distribución, dentro de cada una de las parcelas, conforme a la zonificación por fotointerpretación realizada, encontrando relación entre las zonas con valores muy altos del NDVI con los sectores más vigorosos $\mathrm{y}$ productivos. $\mathrm{Al}$ verse influenciado el valor del NDVI por los elementos del medio, sería recomendable hacer uso del citado índice sobre sectores de las parcelas cuyos factores del medio sean homogéneos.

Tabla 1. Valores de comparación de medias y sus agrupaciones mediante el test de Duncan, así como su significación (sg) para un nivel de probabilidad $\mathrm{p}<0,001(* * *)$ para: Racimos ( $\mathrm{N}^{\mathrm{o}}$ de Racimos), Producción (Racimos*PRacimo), PRacimo (Peso del racimo, gr), Longitud (Longitud del sarmiento, cm), Calibre (Calibre del sarmiento, cm), Pesomad (Peso de madera de poda, gr) y Ravaz (Índice de Ravaz: Pesomad/Producción)

\begin{tabular}{|c|c|c|c|c|c|c|c|}
\hline SECTOR & RACIMOS & PRODUCCIÓN & PRACIMO & LONGITUD & CALIBRE & PESOMAD & RAVAZ \\
\hline A1 & $4 \mathrm{a}$ & $330,5 \mathrm{a}$ & $86,25 \mathrm{abc}$ & $75,5 \mathrm{ab}$ & $0,862 \mathrm{a}$ & $228,7 \mathrm{a}$ & $1,64 \mathrm{ab}$ \\
\hline $\mathrm{A} 2$ & $6,42 \mathrm{ab}$ & $481,17 \mathrm{a}$ & $78,63 \mathrm{abc}$ & $101,25 \mathrm{c}$ & $1,048 \mathrm{~cd}$ & $276,67 \mathrm{ab}$ & $1,89 \mathrm{ab}$ \\
\hline $\mathrm{A} 3$ & $6 \mathrm{a}$ & $552,00 \mathrm{a}$ & $98,10 \mathrm{bc}$ & $77,5 \mathrm{ab}$ & $0,916 \mathrm{ab}$ & $297,5 \mathrm{ab}$ & $1,83 \mathrm{ab}$ \\
\hline $\mathrm{B} 1$ & $5,78 \mathrm{a}$ & $356,78 \mathrm{a}$ & $68,17 \mathrm{ab}$ & $82,33 \mathrm{ab}$ & $0,867 \mathrm{a}$ & $348,44 \mathrm{ab}$ & $0,91 \mathrm{a}$ \\
\hline B2 & $5,6 \mathrm{a}$ & $316,2 \mathrm{a}$ & $56,15 \mathrm{a}$ & $84 \mathrm{ab}$ & $0,851 \mathrm{a}$ & $442,8 \mathrm{bc}$ & $0,76 \mathrm{a}$ \\
\hline C1 & $9,75 \mathrm{bc}$ & $1991,5 \mathrm{~d}$ & $198,25 \mathrm{~d}$ & $122,08 \mathrm{~d}$ & $1,041 \mathrm{~cd}$ & $570,33 \mathrm{c}$ & $3,5 \mathrm{c}$ \\
\hline $\mathrm{C} 2$ & $10,25 \mathrm{c}$ & $1901,33 \mathrm{~cd}$ & $186,23 \mathrm{~d}$ & $107,5 \mathrm{c}$ & $0,993 \mathrm{bcd}$ & $534,67 \mathrm{c}$ & $3,6 \mathrm{c}$ \\
\hline $\mathrm{D} 1$ & $11,92 \mathrm{~cd}$ & $1300,75 \mathrm{~b}$ & $105,08 \mathrm{c}$ & $71,25 \mathrm{a}$ & $1,087 \mathrm{~d}$ & $362,67 \mathrm{ab}$ & $3,57 \mathrm{c}$ \\
\hline $\mathrm{D} 2$ & $18,5 \mathrm{e}$ & $1708,25 \mathrm{bcd}$ & $89,11 \mathrm{bc}$ & $107,08 \mathrm{c}$ & $0,967 \mathrm{abc}$ & $845,5 \mathrm{~d}$ & $2,49 \mathrm{bc}$ \\
\hline $\mathrm{D} 3$ & $14,67 \mathrm{~d}$ & $1339,33 \mathrm{bc}$ & $91,27 \mathrm{bc}$ & $88,33 \mathrm{~b}$ & $0,867 \mathrm{a}$ & $540,17 \mathrm{c}$ & $2,7 \mathrm{bc}$ \\
\hline $\mathrm{sg}$ & $* * *$ & $* * *$ & $* * *$ & $* * *$ & $* * *$ & $* * *$ & $* * *$ \\
\hline
\end{tabular}


Tabla 2. Valores de comparación de medias y sus agrupaciones mediante el test de Duncan, así como su significación (sg) para un nivel de probabilidad $\mathrm{p}<0,05(*), \mathrm{p}<0,01(* *)$ y $\mathrm{p}<0,001(* * *)$ para: Grado (Grado de Alcohol Probable, \% volúmen), A.Total (Acidez

Total, gr/l de Ácido Tartárico), Málico (Ácido Málico, gr/l), I.C. (Índice de Color), I.P.T. (Índice de Polifenoles Totales), Antoc (Antocianos, mg/l), P100Bayas (peso de 100 bayas, gr), Potasio (Potasio, mg/l) y Tartárico (Ácido Tartárico, gr/l)

\begin{tabular}{|c|c|c|c|c|c|c|c|c|c|c|}
\hline SECTOR & GRADO & A.TOTAL & $\mathrm{pH}$ & MALICO & I.C. & I.P.T. & ANTOC & P100BAYAS & POTASIO & TARTÁRICO \\
\hline A1 & $15,84 \mathrm{~d}$ & $3,25 \mathrm{a}$ & $3,93 \mathrm{abcd}$ & $1,95 \mathrm{a}$ & $20,0855 \mathrm{~cd}$ & $67,85 \mathrm{ef}$ & $846,5 \mathrm{c}$ & $144,5 \mathrm{a}$ & $2140 \mathrm{ab}$ & $4,9 \mathrm{a}$ \\
\hline A2 & $15,63 \mathrm{~cd}$ & $3,7 \mathrm{abc}$ & $3,8 \mathrm{abc}$ & $1,35 \mathrm{a}$ & $23,3105 \mathrm{~d}$ & $59,9 \mathrm{de}$ & $835 \mathrm{~cd}$ & $161 \mathrm{ab}$ & $1785 \mathrm{a}$ & $4,3 \mathrm{a}$ \\
\hline A3 & $16,04 \mathrm{~d}$ & $3,6 \mathrm{ab}$ & $4,11 \mathrm{~d}$ & $2,4 \mathrm{a}$ & $22,8640 \mathrm{~d}$ & $72,6 \mathrm{f}$ & $1032,5 \mathrm{e}$ & $130 \mathrm{a}$ & $3240 \mathrm{c}$ & $4,7 \mathrm{a}$ \\
\hline B1 & $15,09 \mathrm{bcd}$ & $4,25 \mathrm{c}$ & $3,99 \mathrm{~cd}$ & $1,85 \mathrm{a}$ & $19,2365 \mathrm{bc}$ & $58,2 \mathrm{~cd}$ & $835,5 \mathrm{~cd}$ & $148 \mathrm{a}$ & $2075 \mathrm{ab}$ & $4,6 \mathrm{a}$ \\
\hline B2 & $15,67 \mathrm{~cd}$ & $3,85 \mathrm{abc}$ & $3,97 \mathrm{bcd}$ & $2,25 \mathrm{a}$ & $19,2740 \mathrm{bc}$ & $59,9 \mathrm{de}$ & $847,5 \mathrm{~d}$ & $150,5 \mathrm{a}$ & $2615 \mathrm{~b}$ & $4,8 \mathrm{a}$ \\
\hline C1 & $14,26 \mathrm{abc}$ & $4,25 \mathrm{c}$ & $3,9 \mathrm{abc}$ & $2,75 \mathrm{a}$ & $14,4460 \mathrm{a}$ & $47,4 \mathrm{ab}$ & $689 \mathrm{abc}$ & $196 \mathrm{~b}$ & $2630 \mathrm{~b}$ & $4,8 \mathrm{a}$ \\
\hline C2 & $14,52 \mathrm{abcd}$ & $3,85 \mathrm{abc}$ & $3,79 \mathrm{ab}$ & $2,15 \mathrm{a}$ & $14,8075 \mathrm{a}$ & $43,25 \mathrm{ab}$ & $620 \mathrm{a}$ & $193 \mathrm{~b}$ & $1945 \mathrm{a}$ & $4,4 \mathrm{a}$ \\
\hline D1 & $13,52 \mathrm{a}$ & $3,85 \mathrm{abc}$ & $3,84 \mathrm{abc}$ & $1,55 \mathrm{a}$ & $16,2510 \mathrm{ab}$ & $52,5 \mathrm{bcd}$ & $777 \mathrm{bcd}$ & $140 \mathrm{a}$ & $2260 \mathrm{ab}$ & $4,5 \mathrm{a}$ \\
\hline D2 & $13,74 \mathrm{ab}$ & $3,6 \mathrm{ab}$ & $3,73 \mathrm{a}$ & $2,35 \mathrm{a}$ & $16,0960 \mathrm{ab}$ & $40,9 \mathrm{a}$ & $636 \mathrm{ab}$ & $102 \mathrm{ab}$ & $1990 \mathrm{a}$ & $4,6 \mathrm{a}$ \\
\hline D3 & $14,56 \mathrm{abcd}$ & $3,9 \mathrm{bc}$ & $3,8 \mathrm{abc}$ & $1,75 \mathrm{a}$ & $18,4295 \mathrm{bc}$ & $49,4 \mathrm{abc}$ & $747,5 \mathrm{abcd}$ & $142 \mathrm{a}$ & $2015 \mathrm{a}$ & $4,8 \mathrm{a}$ \\
\hline sig. & $*$ & $\mathrm{~ns}$ & $*$ & $\mathrm{~ns}$ & $* *$ & $* * *$ & $* *$ & $*$ & $* *$ & $\mathrm{~ns}$ \\
\hline
\end{tabular}

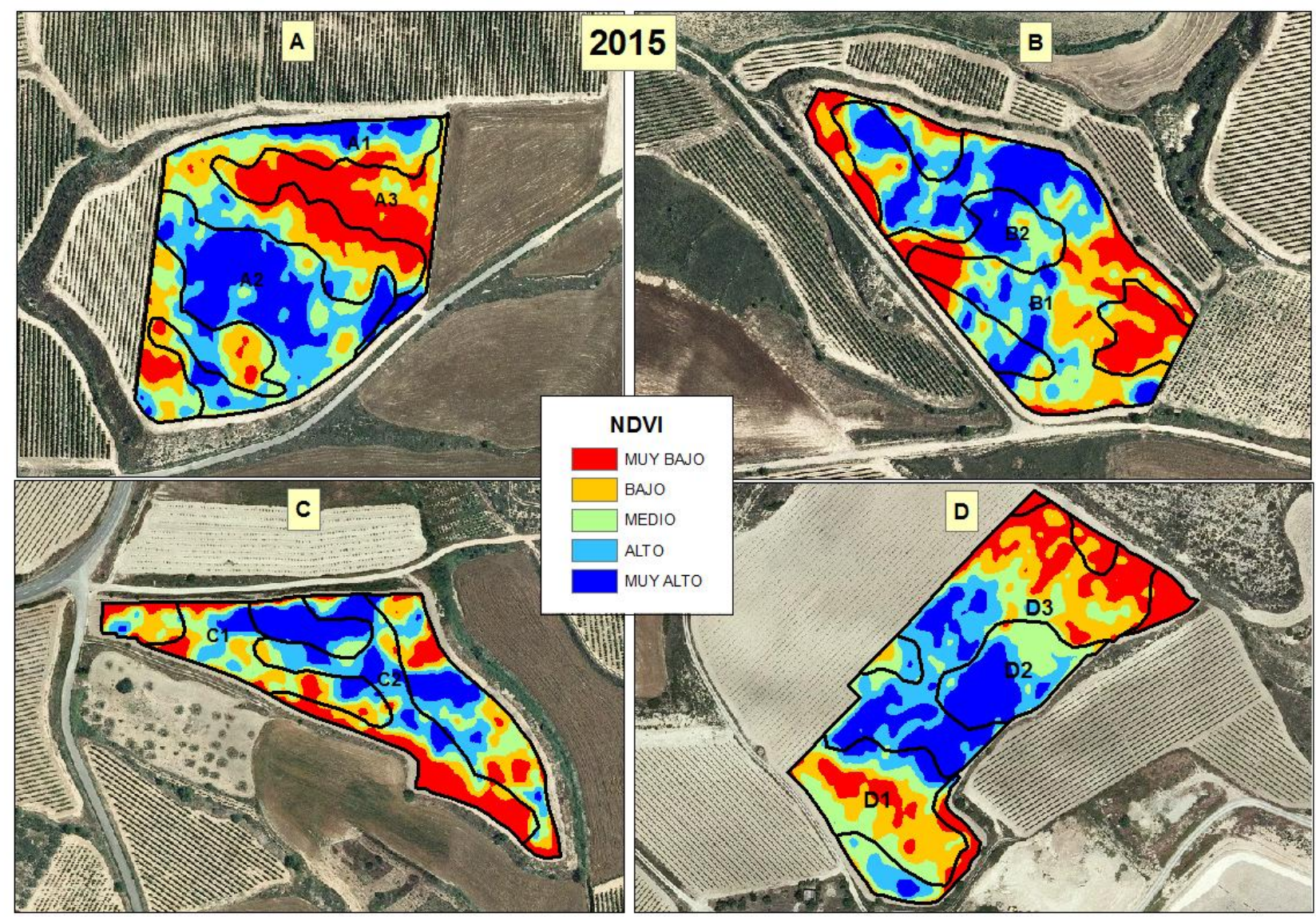

Figura 1. Representación gráfica del NDVI de las parcelas de estudio, calculado pixel a pixel. La clasificación se lleva a cabo conforme a 5 cuantiles y en cada parcela de forma independiente

\section{Referencias}

1. Morlat, R. in Le Terroir viticole: contribution a l'etude de sa caracterisation et de son influence sur les vins, application aux vignobles rouges de moyenne Vallée de la Loire (ed Université de Bordeaux II. Institut d'Oenologie), (fr), 1989).

2. OIV. Definición de terroir vitivinícola. (2010).

3. Pan-Montojo, J. in Mediterráneo e Historia Económica (eds Nadal, J. \& Parejo, A.) (Caja Rural Intermediterránea. Cajamar, Almería, 2005). 
4. Jones, G. V. in Fine wine and terroir: the geoscience perspective (eds Macqueen, R. W., Meinert, L. D. \& Geological Association of Canada) (Geological Association of Canada, St. John's, Canada, 2006).

5. Tonietto, J. \& Carbonneau, A. A multicriteria climatic classification system for grape-growing regions worldwide. Agric. For. Meteorol. 124, 81-97 (2004).

6. Gómez-Miguel, V. in Atlas das castas da Península Ibérica: história, terroir, ampelografia (ed Böhm, J.) 104-153 (Dinalivro, Lisboa, Portugal, 2011).

7. Hancock, J. M. in Encyclopedia of Geology (ed Plimer, R. C. S. R. M. C. R.) 85-89 (Elsevier, Oxford, 2005).

8. Huggett, J. Geology and wine: a review. Proc. Geol. Assoc. 117, 239-247 (2006).

9. Bramley, R. G. V. in Managing wine quality (ed Reynolds, A. G. (. G.) 445-479 (Woodhead; CRC Press, Oxford: Boca Raton: (uk), 2010).

10. van Leeuwen, C. in Managing wine quality. V1: Viticulture and wine quality (ed Reynolds, A. G.) 273315 (Woodhead, Oxford, 2010).

11. Vaudour, E. in Los terroirs vitícolas: definiciones, caracterización y protección (Acribia, D.L., Zaragoza, 2010).

12. Jackson, D. \& Lombard, P. Environmental and Management-Practices Affecting Grape Composition and Wine Quality - a Review. Am. J. Enol. Vitic. 44, 409-430 (1993).

13. van Leeuwen, C. \& Seguin, G. The concept of terroir in viticulture. Journal of Wine Research 17, 1-10 (2006).

14. Gladstones, J. in Wine, Terroir and Climate Change [Recurso electrónico].] (Wakefield Press, Adelaide : (at), 2011).

15. Columela, L. J. M. in Los doce libros de agricultura. (ed Álvarez de Sotomayor y Rubio,Juan María) (Extramuros, Mairena de Aljarafe (Sevilla) : (sp), 2008).

16. Seguin, G. Terroirs and Pedology of Wine Growing. Experientia 42, 861-873 (1986).

17. van Leeuwen, C. et al. Influence of climate, soil, and cultivar on terroir. Am. J. Enol. Vitic. 55, 207-217 (2004).

18. Santesteban, L. G., Guillaume, S., Royo, J. B. \& Tisseyre, B. Are precision agriculture tools and methods relevant at the whole-vineyard scale? Precis. Agric. 14, 2-17 (2013).

19. Witze, A. The grapes of rock. Nature 438, 1073-1074 (2005).

20. Martínez, A., Gomez-Miguel,V. Vegetation index cartography as a methodology complement to the terroir zoning for its use in precision viticulture. OENO One $\mathbf{5 1}$ (2017).

21. Cadet de Vaux, A. in Arte de hacer el vino (Maxtor, Valladolid, 1803).

22. Tisseyre, B., Ojeda, H. \& Taylor, J. New technologies and methodologies for site-specific viticulture. J. Int. Sci. Vigne Vin. 41, 63-76 (2007).

23. Bramley, R. G. V., Ouzman, J. \& Boss, P. K. Variation in vine vigour, grape yield and vineyard soils and topography as indicators of variation in the chemical composition of grapes, wine and wine sensory attributes. Aust. J. Grape Wine Res. 17, 217-229 (2011).

24. Bramley, R. G. V. \& Hamilton, R. P. Terroir and precision viticulture: Are they compatible? Journal
International Des Sciences De La Vigne Et Du Vin 41, 18 (2007).

25. Lamb, D., Weedon, M. \& Bramley, R. Using remote sensing to predict grape phenolics and colour at harvest in a Cabernet Sauvignon vineyard: Timing observations against vine phenology and optimising image resolution. Australian Journal of Grape and Wine Research 10, 4654 (2004).

26. Van Leeuwen, C. et al. Vine Water Status is a Key Factor in Grape Ripening and Vintage Quality for Red Bordeaux Wine. how can it be Assessed for Vineyard Management Purposes? J. Int. Sci. Vigne Vin. 43, 121134 (2009).

27. Chone, X., van Leeuwen, C., Chery, P. \& RibereauGayon, P. Terroir inluence on water status and nitrogen status of non-irrigated Cabernet Sauvignon (Vitis vinifera)Vegetative development, must and wine composition (example of a Medoc top Estate vineyard, Sain Julien Area, Bordeaux, 1997) . South african journal for enology and viticulture 22, 815 (2001).

28. Chone, X. et al. Effect of vine nitrogen status on grape aromatic potential: Flavor precursors (S-cysteine conjugates), glutathione and phenolic content in Vitis vinifera L. cv. Sauvignon blanc grape juice. J. Int. Sci. Vigne Vin. 40, 1-6 (2006).

29. Bramley, R. \& Hamilton, R. Understanding variability in winegrape production systems 1 . Within vineyard variation in yield over several vintages. Australian Journal of Grape and Wine Research 10, 3245 (2004).

30. Bramley, R. Understanding variability in winegrape production systems - 2. Within vineyard variation in quality over several vintages. Aust. J. Grape Wine Res. 11, 33-42 (2005).

31. Unamunzaga, O. Zonificación de un viñedo de Rioja Alavesa según las propiedades del suelo y su influencia sobre producción y calidad. (2010). 